\title{
The Relationship between Interpersonal Trust and Loneliness of University Students: The Mediating Role of Personality Hardiness
}

\author{
Lishen Wang \\ School of Education \\ Shaoguan University \\ Shaoguan City, China \\ 04033008@163.com
}

\begin{abstract}
Objective: To understand the status quo of university students' personality hardiness, interpersonal trust and loneliness, and to explore the relationship between those three attributes. - Methods: A total of $\mathbf{3 3 2}$ university students in Guangdong province were selected as the research objects, and selected personality hardiness measurement scale, interpersonal trust scale and the UCLA Loneliness Scale (the third edition) as research tools.- Results: University students' total level of persistence, responsibility, challenge capability and hardiness, and loneliness, interpersonal trust were significantly different in gender variables ; the levels of persistence and hardiness of university students were significantly different in grade variables ; The responsibility and the capacity of challenge of university students were significantly different in the leadership roles students carried out ; The interpersonal trust and challenge, control and hardiness have shown a significant negative correlation ; Loneliness has shown a significant negative correlation with personality hardiness in every dimension ; Interpersonal trust and loneliness are significantly positive related ; The personality hardiness has a mediating role between interpersonal trust and loneliness, with the effect accounted for $10.9 \%$ of the total effect. Conclusion: The personality hardiness of university students plays a part of mediating role in the relationship between interpersonal trust and loneliness.
\end{abstract}

Keywords—university students; personality hardiness; interpersonal trust; loneliness; mediating role

\section{INTRODUCTION}

Personality hardiness was first used to study the relationship between employee's personality, work stress and health, this concept was first introduced by Kobasa into the field of psychology[1].

Kobasa argues that personality hardiness is a set of attitudes, beliefs, and behaviors that can help people manage stress. Individuals with this trait give life positive and focus on work, and they believe they have the ability to influence what happens around them, and they will have clear purpose to take action on the uncertain things in life, they see change as an opportunity to help individual growth, rather than threaten their own security situation. In Kobasa's view, hardiness is a personality structure that can be used to resist stress. Individuals with this trait can be protected from stress even in a highly stressful stress situation. Rotter (1967) argues that interpersonal trust is the expectation of the degree of reliability of an individual or group to another group of languages, commitments and discourses or written statements [2]. There have been some achievements in the study of interpersonal trust in China, but some issues are still to be developed, such as lack of empirical survey data, lack of theoretical research and conceptual combing, lack of empirical research on interpersonal trust content, and so on. Russell et al. (1988) argue that loneliness will be felt when there is a gap between the expectations of social interaction and the actual level[3]. Because the researchers have different understanding of loneliness, which results in not a recognized concept exist in this field, so the preparation of the measurement scale is different, the conclusions are different. As a result, many studies on loneliness have differences on demographic variables.

Zhou Qian (2011) shows that interpersonal trust can predict loneliness[4], but it is not clear whether there are other influencing variables, so this study use personality hardiness as an intermediary variable to explore whether there is a mediating effect. This study adopts personality hardiness measurement scale, interpersonal trust scale and the UCLA Loneliness Scale to evaluate university students' personality hardiness, interpersonal trust and loneliness, and analyze the relationships between these three attributes. This study will enrich the existing study from an empirical research angle, thus to supplement the existing research for the psychological health education in universities and provide theoretical support.

Sponsored by characteristics of innovation projects from Education Department of Guangdong Province(2014GXJK135) and the key discipline project of Education in Guangdong Province Supported by Education of Shaoguan University. 


\section{OBJECTIVE AND METHOD}

\section{A. Objective}

The university students in Guangdong Province are selected as the research objects. A total of 400 questionnaires were issued and 332 valid questionnaires were collected.

\section{B. Method}

The template We adopted the personality hardiness scale proposed by Lu (2012) [5] which is based on China's national situations. The Scale has 27 projects, including four dimensions, namely, responsibility, controllability, capability of facing challenge and persistence. Responsibility reflects the personality characteristics of individuals focusing on the current activities and take the courage to be responsible; Controllability reflects the characteristics of individuals to take the initiative to control and affect the results of things experienced; Capability of facing challenge refers to the characteristics of individual who dare to challenge the changing situation, and develop their personality; Persistence reflect the characteristics of individuals who firmly in pursuit of their goals and never give up when facing the difficulties. The recalculation reliability of each sub-scale was 0.89-0.92, the internal consistency coefficient $\alpha$ was $0.749-0.853$; The recalculation reliability of the total scale was 0.92 , and the internal consistency $\alpha$ coefficient was $0.927[6]$.

Rotter's Interpersonal Trust Scales (ITS), which are used to measure interpersonal trust in various contexts. This study directly adopted the Chinese translation version Scale in the Mental Health Rating Scale (updated edition) from Wang Xiangdong et al. (1999). The scale consists of total 25 questions and major two factors, namely, general trust (trust for no direct relationship) and special trust (trust in companions or other family members), but the editor does not specify the factors corresponding to each question. The survey uses 5-scale Lickert chart, the scores of the interpersonal trust score are ranging from 25-125 points; The higher the score, the higher the level of interpersonal trust; The 75 points is the dividing line. This Scale has higher discriminant validity, the recalculation reliability is 0.56 [7], can be used to test our national interpersonal trust.

The UCLA Loneliness Scale (third edition), which was compiled by Russell, and edited by Wang Dengfeng, could be used to test the loneliness caused by the disparity between social desire and reality. The Scale has a total of 20 questions, with 4 Likertscale; The total score ranging from 20 points to 80 points; The norm score is 44 points; The higher the score, the individual's lonely experience more intense. The internal consistency coefficient of the scale is 0.93 , the project discriminant validity ranging from 0.55-0.72, and the recalculation reliability with an interval of 7 months is 0.62[7]. Wang Dengfeng's (1995) study has shown that the scale can be used for university students to measure their loneliness, which has a higher conceptual validity and discriminant validity [1].

\section{RESULTS AND DISCUSSION}

\section{A. Demographic Analysis of University Students' Personality Hardiness, Interpersonal Trust and Loneliness}

The descriptive statistics of 332 university students' personality hardiness with multiple dimensions, interpersonal trust and loneliness were analyzed by SPSS.The scores of the four dimensions of the hardiness of university students ranked from high to low as controllability, challenge capability, persistence, and responsibility respectively, ranging from $71.23 \pm 11.69$. The total score of the loneliness scale was $53.99 \pm 7.86$, and the total score of the interpersonal trust scale was 78.49 \pm 7.10 .

The results of this study show that there are significant differences in persistence $(\mathrm{p}=0.003)$, responsibility $(\mathrm{p}=0.026)$, challenge capability $(\mathrm{p}=0.030)$ and total hardiness $(\mathrm{p}=0.017)$ among university students with different genders, which is in line with the research results from Ma Xianming[8] (2008) and Duan Haiyan[9] (2010). The perspectives towards different roles of gender may also lead to differentiated parents' attitude to educate their children. So in the pursuit of goals, the boys may be more determined in their own beliefs than girls, when they will not give up when encountered difficulties. These attitude and education make the male's scores significantly higher than girls.

Whether the student carrying out a student leader role will also lead to significantly difference for responsibility $(\mathrm{P}=0.045)$ and challenge capability $(\mathrm{p}=0.031)$. Students who have served as student leaders have high scores. The students who have shown more willingness to participate in various activities, the courage to challenge to the unknown things are more likely to become student leaders; or it may be because after serving as a student leader, the students have gain diverse values of things, are more willing to complete the challenging work.

There were significant differences in university students' persistence $(\mathrm{p}=0.035)$ and the overall level of personality hardiness $(\mathrm{p}$ $=0.041$ ) in terms of different grades. This finding is similar with the research result of He Jie[10] (2014). This maybe due to the junior students already studied and lived in the university for two years, their personal experience increased a lot, which has benefited their ability in willpower, action, autonomy and the ability to deal with the problem are more developed than the freshman students.

Another finding is that there was a significant difference in loneliness $(p=0.049)$ among students of different genders, which is the same as that of MA Su-hong[11] (2007). The sense of loneliness of the boys is stronger than the girls, perhaps because of the 
different ways of family education, the character traits of the boys and girls are different, girls are more tend to hang out in groups. It may also because of the different ways that boys and girls respond, girls will use a more positive way to deal with events in life and looking for social support, which may reduce the sense of loneliness.

There were significant differences in interpersonal trusts $(p=0.001)$ among different genders, which was the same as that of Tian Kexin[12] (2005). This may be related to the cognitive style differences between boys and girls. It may also because of the parents education attitude towards boys and girls - girls are often educated that they should not talk to strangers, do not easily believe in others, which may lead to girls' interpersonal trust is weaker than boys.

\section{B. Correlation Analysis of University Students' Personality Hardiness, Interpersonal Trust and Loneliness}

The data of 332 university students were statistically analyzed by SPSS. The results show there was a significant negative correlation between interpersonal trust and challenge capability $(\mathrm{p}=0.007)$, controllability $(\mathrm{p}=0.01)$ and the overall level of personality hardiness $(\mathrm{p}=0.02)$.Loneliness was negative correlation with the overall level of persistence, responsibility, challenge capability, controllability, and personality hardiness. The correlation coefficient of interpersonal trust and loneliness was 0.237 ( $\mathrm{p}$ $<0.01$ ), which indicating that interpersonal trust and loneliness were significantly positively correlated.

The results of this study show a significant negative correlation between interpersonal trust and challenge capability $(p=0.007)$, controllability $(\mathrm{p}=0.01)$ and the overall level of personality hardiness $(\mathrm{p}=0.02)$. It may be because of the individuals with high scores in challenge capability, controllability and personality hardiness will tend to contribute more in the activity and try to do things well. Since group collaboration activities will be more prone to social laziness, so they believe in their own ability and feel that a person can do a good job, which is too dependent on their own ideas, and may reduce interpersonal trust.

Loneliness was negatively correlated with the overall level of persistence, responsibility, challenge capability, controllability and personality hardiness, the correlation coefficients were $-0.242,-0.272,-0.281,-0.250$ and -0.291 respectively. There is a significant negative correlation between loneliness and persistence, which may be due to the fact that individuals with high persistence scores tend to exhibit optimism and firmness in the face of difficulties, so they may experience less loneliness. The reason that loneliness is significantly negative correlated with responsibility and challenge capability may be that individuals with higher scores in responsibility and challenge capability may feel highly fulfilled every day and have no time for self-pity, thus, they may experience less loneliness. A significant negative correlation between loneliness and control may be due to the fact that individuals with high levels of controllability scores will positively influence the outcome of the event, and that individuals with positive emotions are more socially supported and less lonely. Loneliness and the level of personality hardiness is likely to be negative related, because the hardiness personality is a positive personality trait, which can prevent stress. Individuals with hardiness personality will adjust proactively to reduce the loneliness in the face of loneliness.

The Pearson correlation coefficient of interpersonal trust and loneliness was 0.237 ( $p<0.01)$, indicating that interpersonal trust and loneliness were significantly positively correlated. This result is similar with the result of Chen Shu [13] (2010) while contrary with Li Longhui et al.'s [14] (2010). The reason for the divergence may be that the trusts and loneliness scales used by the researchers in the study are not the same, which leads to differences in the trust and loneliness scores. Although it is possible to determine the relationship between interpersonal trust and loneliness, the direction of the relationship between interpersonal trust and loneliness needs to be studied in depth.

\section{Regression Analysis of University Students' Personality Hardiness, Interpersonal Trust and Loneliness}

We use SPSS statistical software to conduct the regression analysis of university students' personality hardiness, interpersonal trust and loneliness, the result shows that university students' interpersonal trust and personality hardiness have shown a significant regression result, the both together can explain $1.6 \%$ of the amount of variability of loneliness. the mediating effect model is plotted as shown in Figure 1.

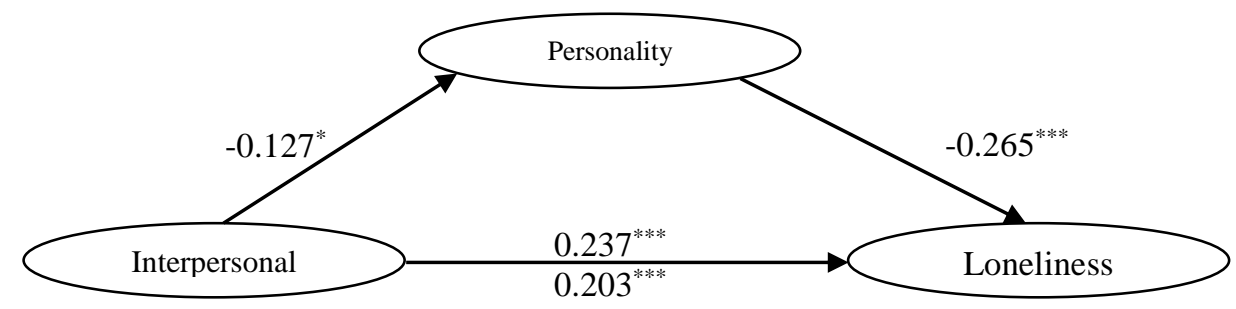

Figure 1 The Mediating Effect Model of Personality Hardiness in the Relationship between Interpersonal Trust and Loneliness

\section{CONCLUSION}

Due to gender differences, there were significant differences of university students' persistence, controllability, challenge capability and the total level of personality hardiness, loneliness and interpersonal trust. The level of persistence and personality 
hardiness of university students was significantly different in grade variables. The level of university students' responsibility and challenge capability was significantly different in the student leader variables. Interpersonal trust was significant negative correlation with challenge capability, control liability and personality hardiness. Loneliness was significant negative correlation with personality hardiness in every dimension. Interpersonal trust and loneliness were significantly positive correlated. Personality hardiness partially played a mediating role in the relationship between interpersonal trust and loneliness.

\section{REFERENCES}

[1] Zou, Z., Wang, D. Stress Buffer: Personality Hardiness [J]. Advances in Psychological Science, 2007,15 (2): $241-248$.

[2] Rotter J B. A new scale for the measurement of interpersonal trust [J]. Journal of Personality. 1967, 35: 651-665.

[3] Russell, D. W. , Cutrona, C. E. Development and evolution of the UCLA Loneliness Scale [J]. Unpublished manuscript, Center for Health Services Research, University of Medicine, University of Lowa, 1988, 21: 51-66.

[4] Zhou, Q., Yao, Z. The relationship study of college students' personality traits, interpersonal trust and loneliness [J]. Chinese Journal of Health Psychology, 2011,19 (9): 1110-1112.

[5] Dai, X., edited. Scale manual for common psychological assessment [M] Beijing: People's Military Medical Press, 2010.07: $301-303$.

[6] Lu, G., Yu, L., Liang, B. Mental health quality evaluation system: The making of Chinese adults' personality hardiness scale [J]. Psychological and Behavioral Research, 2012,10 (5): 321-325.

[7] Wang, X., Wang, X., Ma, H. Mental health assessment scale manual (updated version) [M]. Beijing: China Mental Health Journal, 1999 (12): 87-91.

[8] Ma, X. The research on the relationship of college student's personality, coping style and mental health [D]. Jiangsu: Suzhou University, 2008.

[9] Duan, H., Study on the relationship between college students' personality hardiness, social support and subjective well-being [D]. Shanxi: Northwest University, 2010.

[10] He, J., Parenting style on college student's social adaptation: the mediating role of personality hardiness [D]. Henan: Henan University, 2014.

[11] Ma, S., The study on the relationship of graduate student's loneliness and its attachment type and social support [J]. Jilin: Jilin University, 2007.

[12] Tian, K., Wang, X., Wu, H., Li, X., Effects of grade and gender on interpersonal trust and interpersonal relationship of college students [J]. Chinese Journal of Clinical Rehabilitation, 2005 (24): 82-84.

[13] Chen, S., Zhang, X., A preliminary study on Xuzhou University students' interpersonal trust and loneliness [J]. Journal of Science and Technology, 2010 (3): 139-140.

[14] Li, L., Huang, J., Research on university student's emotional loneliness and interpersonal trust [J] Journal of Langfang Teachers College: Social Science Edition, 2010,26 (6): 116-118. 